\title{
Note sur la symétrie inverse des polyèdres non eulériens.
}

\author{
(Par M. Camille Jordan à Paris.)
}

Dans une note insérée au T. 66 de ce journal nous avons indiqué la manière de répartir les surfaces polyédriques en espèces correspondant aux diverses valeurs qui peuvent être assignées à deux paramètres $m$ et $n$ : nous avons en outre indiqué que les polyèdres de l'espèce $(0,1)$ (ceux qui présentent l'aspect général d'un tore, par exemple) sont susceptibles de trois sortes de symétrie directe, que nous avons appelées

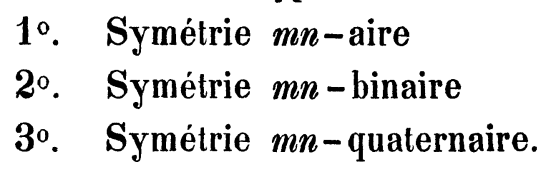

Les paramètres $m$ et $n$ pouvant chacun prendre toutes les valeurs entières possibles, sauf dans le dernier cas, où nous avons signalé une restriction à laquelle ces valeurs sont soumises.

En reprenant récemment la question, je viens de reconnaître, que la restriction indiquée au lieu cité n'est pas suffisante, et qu'on doit avoir nécessairement $n=m$, de sorte que la dénomination de symétrie $m n$-quaternaire doit être remplacée par la suivante: symétrie $m^{2}$-quaternaire. La valeur du paramètre $m$ peut d'ailleurs être un entier quelconque.

D'autre part, en appliquant la méthode exposée dans le mémoire cidessus à la recherche des symétries inverses de ces polyèdres, on obtient les résultats suivants.

Si un polyèdre d'espèce $(0,1)$, et présentant la symétrie $m n$-aire, est en outre inverse à lui-même, trois cas pourront se présenter:

10. Aucun élément ni arête n'est son propre inverse.

Soient dans ce cas $A, \alpha$ deux sommets inverses l'un de l'autre, et choisis aussi voisins que possible, $L$ une ligne géodésique tracée entre $A$ et $\alpha, L, L^{\prime}$ etc. les diverses lignes pareilles ou inverses à $L$ que l'on peut tracer sur le polyèdre. L'ensemble de ces lignes constituera $m$ contours fermés $K, K^{\prime} \ldots$ ne se coupant pas eux-mêmes ni mutuellement: sur chacun de ces contours se trouveront $n$ sommets pareils à $A$, et $n$ pareils à $\alpha$, qui se succèdent, alter- 
nativement. Ces contours découperont enfin la surface du polyèdre en $m$ régions $\boldsymbol{R}, \boldsymbol{R}^{\prime}$ annulaires, pareilles entre elles, et analogues à celles que $m$ cercles méridiens détermineraient sur la surface d'un tore. Chacune de ces régions $R$ est inverse à elle-même, et pareille à elle-même sous $n$ aspects. Soit $A$ une ligne tracée sur l'une des régions $R$ de manière à être la plus courte possible tout en joignant entre eux deux sommets inverses $B$ et $\beta$. Les diverses lignes pareilles ou inverses à $\Lambda$ que l'on peut tracer sur la dite région forment un contour fermé $I$, ne se coupant pas lui-même, et sur lequel se trouveront alternativement $n$ sommets pareils à $B$ et $n$ sommets pareils à $\beta$. Ce contour $I$ partagera l'anneau $R$ en deux anneaux partiels $\rho$ et $\rho^{\prime}$ inverses l'un de l'autre et dont chacun est pareil à lui-même sous $n$ aspects. Enfin si $n$ ne se réduit pas à l'unité, on peut tracer de l'un à l'autre des deux contours qui limitent l'un de ces anneaux, tel que $\varrho, n$ lignes géodésiques $\lambda, \lambda^{\prime}, \ldots$ ne se coupant pas ellesmêmes ni mutuellement et partageant l'anneau en $n$ régions quadrangulaires pareilles entre elles, et qui prises isolément ne présentent plus aucune symétrie.

$2^{\circ}$ et $3^{\circ}$. Il existe des éléments ou arêtes qui sont leurs propres inverses.

Soit $A$ l'un de ces éléments: traçons toutes les zônes $Z, Z^{\prime}$ formées d'éléments inverses à eux-mêmes et pareilles à celles qui passent par cet élément. Elles seront en nombre $m$, ne se couperont elles-mêmes ni mutuellement, passeront chacune par $m$ éléments pareils à $A$ et partageront la surface du polyèdre en $m$ régions $R, \boldsymbol{R}^{\prime}$ annulaires et pareilles entre elles. Chacune de ces régions $R$ est inverse à elle-même, et pareille à elle-même sous $n$ aspects.

Si les régions $R$ ne contiennent aucun élément qui soit son propre inverse, ce qui constituera le second cas, on pourra tracer dans chacune d'elles un contour fermé $I$ la partageant en deux anneaux partiels $\varrho, \rho^{\prime}$ inverses l'un de l'autre: enfin si $n>1$, chacun d'eux pourra être décomposé en $n$ régions quadrangulaires pareilles par des lignes géodésiques joignant ensemble la zône et le contour qui le limitent respectivement.

Si les régions $R$ contiennent des éléments qui soient leurs propres inverses, ces éléments formeront dans chacune d'elles une zône $Y$, qui la partage encore en deux anneaux partiels $\rho, \rho^{\prime}$ inverses l'un de l'autre et décomposables chacun en $n$ régions quadrangulaires pareilles par des lignes géodésiques joignant ensemble les deux zônes qui le limitent.

Passons aux polyèdres d'espèce $(0,1)$ présentant la symétrie $m n$-binaire. Cinq cas seront à distinguer. 
$4^{\circ}$. Si aucun élément n'est inverse à lui-même, on pourra tracer, comme dans le $1^{\text {er }}$ cas de la symétrie $m n$-aire, $2 m$ contours fermés $K, K^{\prime}, \ldots$, $I$ etc. partageant le polyèdre en $2 m$ régions annulaires $\varrho, \varrho^{\prime}, \ldots$ dont chacune sera l'inverse de ses voisines, et sera sa propre homologue sous $2 n$ aspects différents. Des lignes géodésiques en nombre $2 n$ convenablement tracées d'un bord à l'autre de chacun de ces anneaux le partagent en $2 n$ régions quadrangulaires pareilles de deux en deux. Chacune de ces régions sera sa propre homologue sous deux aspects différents et contiendra en son intérieur un des éléments binaires (ou des arêtes à retournement) que renferme le polyèdre.

$5^{\circ}$ et $6^{\circ}$. Supposons qu'aucun des éléments binaires (ou des arêtes à retournement) ne soit inverse à lui-même, mais qu'il existe d'autres éléments inverses à eux-mêmes.

Soient $A$ l'un de ces éléments, $Z, Z^{\prime}, \ldots$ les zônes en nombre $m$ qui passent par $A$ et ses homologues: elles partagent le polyèdre en $m$ régions annulaires $R, R^{\prime}, \ldots$ qui pourront être subdivisées chacune en deux régions $1^{\circ}$. par un contour fermé tel que $I$ si elles ne contiennent dans leur intérieur aucun élément qui soit son propre inverse: $2^{\circ}$. par une nouvelle zône telle que $\boldsymbol{Y}$ dans le cas contraire. Dans l'un et l'autre cas, le polyèdre sera partagé en $2 m$ régions $\varrho, \varrho^{\prime}, \ldots$ dont chacune est l'inverse de ses voisines, et qu'on peut partager comme dans le cas précédent en $2 n$ régions quadrangulaires, pareilles deux à deux, et dont chacune est sa propre homologue sous deux aspects différents.

7०. Supposons que parmi les quatre systèmes $A, B, C, D$ d'éléments binaires (ou d'arêtes à retournement) il en existe deux, $A$ et $B$, inverses à eux-mêmes, les deux autres, $C$ et $D$, étant inverses l'un de l'autre. Les éléments inverses à eux-mêmes formeront deux systèmes de zônes se croisant sur la surface du polyèdre comme les méridiens et les parallèles d'un tore. Ces systèmes contiendront respectivement $m$ et $2 n$ zônes. Les croisements successifs d'une zône de l'un des systèmes avec celles de l'autre système se feront alternativement suivant des éléments appartenant alternativement à l'espèce $A$ et à l'espèce $B$. Les $2 m n$ régions quadrangulaires sont de deux espèces distinctes, réparties comme les cases blanches et noires sur un échiquier; chacune d'elles contient en son intérieur un élément de l'espèce $C$ ou de l'espèce $D$, et sera homologue à elle-même sous deux aspects diffẻrents.

On voit d'ailleurs aisément que pour que le cas ci-dessus puisse se présenter, il faut nécessairement que m soit pair. 
$8^{\circ}$. Si chacun des 4 systèmes $A, B, C, D$ est son propre homologue, les éléments inverses à eux-mêmes formeront quatre systèmes de zônes: 10. Un système $S$ de $m$ zônes méridiennes passant chacune par $n$ éléments $A$ et $n$ éléments $B$ qui se succèdent alternativement sur son pourtour. $2^{\circ}$. Un second système $S_{1}$ de $m$ zônes méridiennes respectivement situées dans l'intervalle des précédentes et passant chacune par $n$ éléments $C$ et $n$ éléments $D$. $3^{\circ}$. Un système $S_{2}$ de $n$ zônes parallèles coupant celles des systèmes $S$ et $S_{1}$ suivant des éléments $A$ et $C$. $4^{\circ}$. Un système $S_{3}$ de $n$ zônes parallèles, intermédiaires entre les précédentes, et coupant les zônes $S$ et $S_{1}$ suivant des éléments $B$ et $D$. Les 4 systèmes de zônes ci-dessus partagent le polyèdre en $4 m n$ régions quadrangulaires ayant chacune 4 sommets appartenant respectivement à chacuné des 4 espèces $A, B, C, D$. Chacune de ces régions est entièrement dissymétrique : elles sont d'ailleurs ici encore de deux espèces distinctes, réparties comme les cases blanches et noires d'un échiquier.

$9^{\circ}$. Passons enfin aux polyèdres d'espèce $(0,1)$ présentant la symétrie $m^{2}$-quaternaire. Soient $A$ le système d'éléments binaires, $B$ et $C$ les deux systèmes d'éléments quaternaires. Chacun de ces trois systèmes sera inverse à lui-même et les éléments inverses à eux-mêmes se trouveront répartis sur 3 systèmes de zônes dont le premier $S$ est formé de $m$ zônes méridiennes et $m$ zônes parallèles se coupant mutuellement suivant les éléments $B$. Le second système $S_{1}$ est également formé de $m$ zônes méridiennes et $m$ zônes parallèles se coupant mutuellement suivant les éléments $C$ et traversant les précédentes aux éléments $A$. Ces deux systèmes de zônes $S$ et $S_{1}$ partagent le polyèdre en $4 m^{2}$ régions quadrangulaires. A deux sommets opposés de chacune d'elles se trouveront un élément $B$ et un élément $C$, aux deux autres sommets des éléments $A$. Le troisième système $S_{2}$ est formé de $2 m$ zônes traversant diagonalement les régions précédentes de manière à couper les zônes $S$ et $S_{1}$ aux éléments $B$ et $C$. Les régions différentes ainsi déterminées par les trois systèmes de zônes $S, S_{1}, S_{2}$ seront triangulaires en nombre $8 m^{2}$ : aux trois sommets de chacune d'elles seront situés un élément $A$, un élément $B$ et un élément $C$ : enfin deux régions contiguës de part et d'autre d'une même zône seront inverses l'un de l'autre.

Paris, 1868. 